\title{
Effects of environmental enrichment and sexual dimorphism on the expression of cerebellar receptors in C57BL/6 and BTBR +ltpr3tf/J mice
}

\section{Daniela Monje-Reyna}

The University of Texas at San Antonio

Jorge Manzo Denes

Universidad Veracruzana

Fidel Santamaria ( $\nabla$ fidel.santamaria@utsa.edu )

The University of Texas at San Antonio https://orcid.org/0000-0001-8750-2932

\section{Research Article}

Keywords: synaptic receptors, excitability, behavior, plasticity, multi-photon imaging, autism spectrum disorders.

Posted Date: February 18th, 2022

DOI: https://doi.org/10.21203/rs.3.rs-1140363/v1

License: (c) (i) This work is licensed under a Creative Commons Attribution 4.0 International License. Read Full License 


\section{Abstract}

Objective: Environmental enrichment is used to treat social, communication, and behavioral deficits and is known to modify the expression of synaptic receptors. We compared the effects of environmental enrichment in the expression of glutamate and endocannabinoid receptors, which are widely expressed in the cerebellar cortex. These two receptors interact to regulate neuronal function and their dysregulation is associated with behavioral changes. We used BTBR +Itpr3tf/J mice, a strain that models behavioral disorders, and C57BL/ 6 mice for comparison. We studied the effects of genetic background, sex, environmental conditions, and layer of the cerebellar cortex on the expression of each receptor.

Results: The influence of genetic background and environmental enrichment had the same pattern on glutamate and endocannabinoid receptors in males. In contrast, in females, the effect of environmental enrichment and genetic background were different than the ones obtained for males and were also different between the glutamate and endocannabinoid receptors. Furthermore, an analysis of both receptors from tissue obtained from the same animals show that their expression is correlated in males, but not in females. Our results suggest that environmental enrichment has a receptor dependent and sexual dimorphic effect on the molecular expression of different receptors in the cerebellar cortex.

\section{Introduction}

The BTBR+ltpr3tf/J (BTBR) mouse model is widely used in studies related to social and communication deficits, and repetitive behaviors (1-3). BTBR mice exposed to environmental enrichment show decreases in repetitive behaviors and anxiety $(4,5)$, and show an increase in social affiliation (6). In separate studies at the molecular level, changes in the expression of NMDAR1s $(7,8)$ and CB1Rs $(9)$ are modulated by environmental enrichment. Interactions between NMDAR1s and CB1Rs, contribute to regulate neuronal function $(10,11)$, including in the cerebellum (12) where they are widely expressed in the cerebellar cortex $(13,14)$. For these reasons, we quantified the expression of NMDAR1s and CB1Rs in the cerebellar cortex. In particular, we measured these changes in lobule VII because changes in the structure and physiology of this area correlate with abnormal behaviors such as compulsive rituals, stereotypical performance, and difficulty to understand social cues (15), which are replicated in the abnormal behavioral phenotype of the BTBR strain $(16,17)$. The BTBR and C57 groups of each sex were exposed to a standard or enriched environmental conditions. Our results suggest that environmental enrichment has a receptor dependent and sexual dimorphic effect in the cerebellar cortex.

\section{Methods}

\section{Animal procedures}

We bred a new colony for 4 months of C57 (stock \#000664) and BTBR (stock \#002282) mice (Jackson Laboratories, Farmington, CT). Animals were maintained on a 12 hours light/dark-cycle with constant access to food and water. After weaning, animals were separated by sex and housed with their litter in 
standard caging (26 cm width $\times 16 \mathrm{~cm}$ long $\times 16 \mathrm{~cm}$ deep) until P75. Reaching this age, animals from the same litter were randomly selected and separated in those that stayed in the standard environment (standard caging all the time) and those that experienced the environmental enrichment protocol.

The environmental enrichment arena was $90 \mathrm{~cm}$ width $\times 40 \mathrm{~cm}$ long $\times 33 \mathrm{~cm}$ deep and contained dust free bedding (Sophresh Natural Aspen) and toys. The toys were balls, cubes, and pyramids of different sizes, shapes, colors, and textures (solid, hollow, furry); there was also a running wheel, and tunnels. The position of all the toys was changed every day. Animals were placed in the arena 1 hour a day for 20 days during the second four hours of the light part of their light cycle. Animals were returned to their standard cages after each exposure to environmental enrichment. At the end of the 20 days period the standard and environmental enrichment animals were euthanized. We anesthetized mice with $4 \mathrm{~mL}$ isoflurane in an evaporation chamber and kept them in deep anesthesia using the nose cone method. Animals were transcardially perfused with saline followed by $4 \%$ paraformaldehyde $(0.2 \mathrm{M}$ phosphate buffer, $\mathrm{pH} 7.4)$.

\section{Imaging and data analysis}

A detailed description of the immunofluorescence protocol is in the Supplementary Materials. We visualized expression of NMDAR1, CB1R, and DAPI with a multi-photon microscope (Bruker Ultima, Madison, WI). All images were collected with a 20x objective. The wavelength of the tunable laser (DeepSee 690-1300nm, Spectr Physics) to excite at the same time DAPI-NMDAR1 was $1120 \mathrm{~nm}$ and for DAPI-CB1R was $1078 \mathrm{~nm}$. Fluorescent signals were separated by a dichroic cube and detected by photo multipliers. In all cases the scanning laser spent $2.0 \mu \mathrm{s}$ in each coordinate of the image. Images for Figure 1 were background corrected, thresholded, contrast enhanced, and convolved with a Gaussian filter to enhance morphological features. All images used for statistical analyses were only background corrected.

We selected ROIs for the molecular, Purkinje, and granular layers for each receptor in every slice. In the molecular and granular layers, the ROIs were polygons with and average area of $13,072 \mu \mathrm{m}^{2}$ with a rage from 1,718 to $45,365 \mu \mathrm{m}^{2}$ for the granular and $7,098 \mu \mathrm{m}^{2}$ with a range of 1,273 to $22,125 \mu \mathrm{m}^{2}$ for the molecular layer. The ROI for the Purkinje cells were squares of $19.7 \mu \mathrm{m}$ side. We collected, in average, 9.5 Purkinje cell somas per slice with a range from 5 to 15 (see Supplementary Material Figure S1 for an example of ROIs). The ROIs and background correction areas were identical for the receptor and DAPI images. In each ROI we computed a mask of the pixels that contained fluorescent information and used it to calculate the average value of the signal. We then calculated the ratio of NMDAR1/DAPI and CB1R/DAPI. Other groups have successfully used this technique to image brain slices and organoids (18).

For each animal we collected 3 images. The slices were selected based on a clear display of DAPI staining. Each experimental group consisted of 3 animals. No data points were excluded. Confounders were not controlled (such as animal/cage location). Images were analyzed blindly. We used a sample size and power test (sampsizepwrfunction in Matlab) to calculate the minimum number of animals necessary to have a statistical power of $80 \%$. Based on our experimental measurements, the average 
standard deviation in each experimental group was 0.54 . The number of animals required to have at least a difference of 2 in the expression of the receptors with this standard deviation was 3 . We applied a Lilliefors test for all the data and then performed ANOVA and post-hoc tests as described in the results. In all cases we set significance at $\mathrm{p} \otimes 0.05$. We also estimated the effect size of the ANOVA test using the value of $\eta^{2}=\frac{\text { SumSquaresEffect }}{\text { SumSquaresEffect }+ \text { SumSquaresError }}$; for post hoc analyses we used Cohens' d, $d=\frac{\text { Meangroup } 1-\text { MeanGroup } 2}{\text { PopulationStandardDeviation }}(19)$. All the analyses were performed using custom MATLAB scripts (Natick, MA).

\section{Results}

We found NMDAR1s present throughout the cerebellar cortex. NMDAR1s in Purkinje cell somas, dendrites of the molecular layer, and granular cell layer (Figures 1A-D). We found CB1Rs expression on pre-synaptic terminals around Purkinje cell somas, consistent with their presence in the pinceau (Figures 1E-H). There was also diffuse expression in the molecular layer, corresponding to parallel fibers (20). In the granule cell layer the expression corresponded to granule cells somas and dendrites (21).

We performed a multi-way ANOVA test on the expression patterns of each receptor. The test compared genetic background (C57 vs BTBR), sex (male vs female), environmental condition (standard vs enriched caging), and layer in the cerebellar cortex (molecular, Purkinje, and granular). In the case of NMDAR1/DAPI, this test showed that sex was the only significant effect ( $F(1,32)=56.95, p=1.71 \times 10^{-10}$, and the effect size was $\left.\square^{2}=0.44\right)$. The same test for the expression of CB1R showed that sex (sex: $\left.F(1,32)=11.09, p=14.00 \times 10^{-4}, \square^{2}=0.11\right)$ and environmental condition $\left(F(1,32)=16.75, p=1.00 \times 10^{-4}, \square^{2}=0.17\right)$ had a significant effect.

Since sex has a strong influence on the expression of both, NMDAR1 and CB1R, we performed another multi-way ANOVA separating the groups by sex. For NMDAR1 this shows that, for both males and females, the genetic background had a significant effect on the expression of this receptor (males: $F(1,32)=13.73, p=8.00 \times 10^{-4}, \square^{2}=0.22$; females: $\left.F(1,32)=26.69, p=1.33 \times 10^{-5}, \square^{2}=0.22\right)$. This was also the case for the environmental condition (males: $F(1,32)=15.98, p=3.00 \times 10^{-4}, \square^{2}=0.25$; females:

$\left.F(1,32)=56.94, p=1.66 \times 10^{-8}, \square^{2}=0.48\right)$. We obtained the same result when performing the test for the CB1R images (Genetic background: males, $F(1,32)=14.91, p=5.00 \times 10^{-4}, \square^{2}=0.23$; females, $F(1,32)=14.40, p=6.00 \times 10^{-4}, \square^{2}=0.27$; Environmental condition: males, $F(1,32)=18.21, p=1.00 \times 10^{-4}, \square^{2}=0.28$; females, $\left.F(1,32)=6.27, p=0.01, \square^{2}=0.11\right)$.

Next, we performed a post-hoc multi-compare analysis of the multi-way ANOVA tests (groups separated by sex) to determine differences in the effect of environmental enrichment and genetic background in the expression of each receptor. In males, the expression of NMDAR1/DAPI in the BTBR enriched environment group was more than 5 times larger (and significantly different, mean 6.42) than for all the other experimental groups (C57 standard and enriched environment groups, and the BTBR standard environment group, Figure 2A). The same analysis for expression of NMDAR1 in females shows that the 
effect of environmental enrichment is similar for the C57 and BTBR groups. In both cases, the enriched environment had a higher expression of the receptor than the corresponding standard environment group. Thus, suggesting that the genetic background does not modify the effect of environmental enrichment in females (Figure 2B).

We also performed a post-hoc analysis for the CB1R data. This shows that the expression of CB1R in males is identical to the pattern we found for NMDAR1 males (Figure 2C). The expression in females was different from CB1R males and from NMDAR1 males or females. The BTBR groups showed a lower ratio of CB1R/DAPI than the $\mathrm{C} 57$ groups. Environmental enrichment had no effect in changing the expression of CB1R (Figure 2D).

Since we used tissue from the same animals to stain for CB1R or NMDAR1 we studied the relative expression of these receptors. We calculated the correlation coefficient between these values across all animals. We found that the correlation coefficient for all males was large and significant ( $r=0.89, p=1.10 \times 10^{-4}$ ). In contrast, the correlation coefficient for females was not significant ( $r=0.10, p=0.77)$. This analysis suggests a differential regulation in the co-expression of NMDAR1 and CB1R between males and females.

\section{Discussion}

We demonstrated that environmental enrichment affects the expression of NMDAR1s and CB1Rs in vermal lobule VII of the cerebellar cortex. Our results show differences that depended on sex and receptor type, suggesting a complex effect of environmental enrichment in receptor expression in the cerebellar cortex. These findings contribute to the use of the BTBR strain to study sexual dimorphism in neurological disorders in humans (22-24).

While there is no single environmental enrichment paradigm, in all protocols as in this study, the arena is larger than the standard cage and there is a constant reorganization of "toys" (25-29). In our protocol, the session duration for environmental enrichment exposure was based on therapies used in human patients with $\operatorname{ASD}(30,31)$. These environmental therapies change the individual's experience by sensorimotor stimulation, self-directed patterns of attention, and social learning $(32,33)$. Our protocol reproduced these features by the daily change in textures, shapes, sizes, and location of objects, and the mouse-mouse interaction inside the arena. As such, our arena provided an enhanced sensory, motor, social, and cognitive stimulation which meets the criteria in the implementation of this paradigm (29).

Glutamate and endocannabinoid receptors and their pathways can be therapeutic targets to treat abnormal behaviors $(2,34-37)$. In the BTBR mouse NMDA agonist and antagonist have been used to improve sociability and spontaneous grooming $(1,38)$. The activation of endocannabinoid production by acetaminophen enhances social behavior in BTBR mice (39). BTBR animals have a mutation in the Kmo and Ext1 genes, involved in the production of a glutamate receptor antagonist and excitatory synaptic transmission $(40,41)$. It is possible that the increased expression of NMDAR1 in male BTBR mice found 
in our work, is a compensation for the dysregulation in the function of the proteins encoded by these genes, which could have sex differences (24).

\section{Limitations}

Our research would benefit from quantification of changes in behavior to correlate with our molecular expression measurements. A limitation in the interpretation of our results for translational applications in ASD is that while the BTBR mouse model has a strong construct validity it may not reproduce behavioral and cellular symptoms reported in human patients. In addition, data from ASD adult human postmortem brains is not available, and neither data from ASD adult after environmental enrichment, so our data cannot be contrasted.

Our study would benefit from a larger sample size. Particularly, to study small differences between the standard housing groups. However, our current results have a statistical power of $80 \%$ and the effect size shows that the magnitude of the experimental effect is medium to large (42) for the receptor expression.

We concentrated our measurements in Lobule VII because of the known effects of ASD in this area; future work should compare changes in expression across the cerebellum. As an additional limitation the two genotypes used in this work were not littermates, therefore there could be further genetic differences introduced de novo.

\section{Abbreviations}

ASDs

Autism Spectrum Disorders.

BTBR

BTBR +ltpr3tf/J strain mice.

NMDR1s

$\mathrm{N}$-methyl-D-aspartate receptors subunit1.

CB1Rs

endocannabinoid receptors type1.

C57

C57BL/6 strain mice group.

BT

BTBR +ltpr3tf/J strain mice group.

$S$

Standard.

E

Enriched.

ROls

Regions of interest. 
LARC

Laboratory Animal Resources Center.

UTSA

University of Texas at San Antonio .

PBS

Phosphate Buffer Saline.

TBS

Tris-buffered saline.

DAPI

4', 6-diamidino-2-phenylindole.

ANOVA

Analysis of variance.

Env

Environment.

Gen

Genetic background.

$\mathrm{M}$

Male.

$\mathrm{F}$

Female.

$\mathrm{P}$

postnatal day.

Declarations

Ethics approval and consent to participate

All animal procedures were in compliance with the UTSA IACUC and LARC (protocol MU113).

\section{Consent to publish}

Not applicable

\section{Availability of data and materials}

All data and analysis code are available at github.com/Santamarialab/BTBR and upon request.

\section{Competing interests}


The authors declare that they have no competing interests.

\section{Funding}

Contex (UT System-CONACYT) and NIMH-NIBIB 1R01EB026939.

\section{Author contributions}

DM: performed experiments; analyzed data; wrote manuscript. JM: conceptualization and funding. FS: conceptualization; data analysis; wrote manuscript; funding.

\section{Acknowledgments}

Not applicable

\section{References}

1. Burket JA, Benson AD, Tang AH, Deutsch SI. D-Cycloserine improves sociability in the BTBR T+ Itpr3tf/J mouse model of autism spectrum disorders with altered Ras/Raf/ERK1/2 signaling. Brain research bulletin. 2013;96:62-70.

2. Meyza KZ, Blanchard DC. The BTBR mouse model of idiopathic autism - Current view on mechanisms. Neurosci Biobehav Rev. 2017;76(Pt A):99-110.

3. Pearson BL, Pobbe RL, Defensor EB, Oasay L, Bolivar VJ, Blanchard DC, et al. Motor and cognitive stereotypies in the BTBR T+tf/J mouse model of autism. Genes Brain Behav. 2011;10(2):228-35.

4. Reynolds S, Urruela M, Devine DP. Effects of environmental enrichment on repetitive behaviors in the BTBR T+tf/J mouse model of autism. Autism research: official journal of the International Society for Autism Research. 2013;6(5):337-43.

5. Woo CC, Donnelly JH, Steinberg-Epstein R, Leon M. Environmental enrichment as a therapy for autism: A clinical trial replication and extension. Behavioral neuroscience. 2015;129(4):412-22.

6. Diamond BA. Environmental Enrichment Improves Sociability in BTBR Mice, a Rodent Model. for Autism: Syracuse University; 2019.

7. Huang Y, Jiang H, Zheng Q, Fok AHK, Li X, Lau CG, et al. Environmental enrichment or selective activation of parvalbumin-expressing interneurons ameliorates synaptic and behavioral deficits in animal models with schizophrenia-like behaviors during adolescence. Molecular Psychiatry. 2021:120. 
8. Wang X, Meng Z-X, Chen Y-Z, Li Y-P, Zhou H-Y, Yang M, et al. Enriched environment enhances histone acetylation of NMDA receptor in the hippocampus and improves cognitive dysfunction in aged mice. Neural Regeneration Research. 2020;15(12):2327.

9. El Rawas R, Thiriet N, Nader J, Lardeux V, Jaber M, Solinas M. Early exposure to environmental enrichment alters the expression of genes of the endocannabinoid system. Brain research. 2011;1390:80-9.

10. Rodríguez-Muñoz M, Sánchez-Blázquez P, Merlos M, Garzón-Niño J. Endocannabinoid control of glutamate NMDA receptors: the therapeutic potential and consequences of dysfunction. Oncotarget. 2016;7(34):55840-62.

11. Sánchez-Blázquez P, Rodríguez-Muñoz M, Garzón J. The cannabinoid receptor 1 associates with NMDA receptors to produce glutamatergic hypofunction: implications in psychosis and schizophrenia. Front Pharmacol. 2014;4:169-.

12. Netzeband JG, Conroy SM, Parsons KL, Gruol DL. Cannabinoids enhance NMDA-elicited Ca2+ signals in cerebellar granule neurons in culture. The Journal of neuroscience: the official journal of the Society for Neuroscience. 1999;19(20):8765-77.

13. Kawamura Y, Fukaya M, Maejima T, Yoshida T, Miura E, Watanabe M, et al. The CB1 cannabinoid receptor is the major cannabinoid receptor at excitatory presynaptic sites in the hippocampus and cerebellum. The Journal of neuroscience: the official journal of the Society for Neuroscience. 2006;26(11):2991-3001.

14. Lonchamp E, Gambino F, Dupont JL, Doussau F, Valera A, Poulain B, et al. Pre and post synaptic NMDA effects targeting Purkinje cells in the mouse cerebellar cortex. PLoS One. 2012;7(1):e30180.

15. Riva D, Annunziata S, Contarino V, Erbetta A, Aquino D, Bulgheroni S. Gray matter reduction in the vermis and CRUS-II is associated with social and interaction deficits in low-functioning children with autistic spectrum disorders: a VBM-DARTEL Study. Cerebellum. 2013;12(5):676-85.

16. Chao OY, Marron Fernandez de Velasco E, Pathak SS, Maitra S, Zhang H, Duvick L, et al. Targeting inhibitory cerebellar circuitry to alleviate behavioral deficits in a mouse model for studying idiopathic autism. Neuropsychopharmacology: official publication of the American College of Neuropsychopharmacology. 2020;45(7):1159-70.

17. Xiao R, Zhong H, Li X, Ma Y, Zhang R, Wang L, et al. Abnormal Cerebellar Development Is Involved in Dystonia-Like Behaviors and Motor Dysfunction of Autistic BTBR Mice. Frontiers in cell developmental biology. 2020;8:231.

18. Smyrek I, Stelzer EH. Quantitative three-dimensional evaluation of immunofluorescence staining for large whole mount spheroids with light sheet microscopy. Biomedical optics express. 2017;8(2):48499.

19. Lakens D. Calculating and reporting effect sizes to facilitate cumulative science: a practical primer for t-tests and ANOVAs. Frontiers in psychology. 2013;4:863.

20. Safo PK, Regehr WG. Endocannabinoids control the induction of cerebellar LTD. Neuron. 2005;48(4):647-59. 
21. Egertová M, Cravatt BF, Elphick MR. Comparative analysis of fatty acid amide hydrolase and $\mathrm{cb}(1)$ cannabinoid receptor expression in the mouse brain: evidence of a widespread role for fatty acid amide hydrolase in regulation of endocannabinoid signaling. Neuroscience. 2003;119(2):481-96.

22. Baron-Cohen S, Knickmeyer RC, Belmonte MK. Sex differences in the brain: implications for explaining autism. Science. 2005;310(5749):819-23.

23. Schumann CM, Sharp FR, Ander BP, Stamova B. Possible sexually dimorphic role of miRNA and other sncRNA in ASD brain. Molecular autism. 2017;8:4.

24. Werling DM, Parikshak NN, Geschwind DH. Gene expression in human brain implicates sexually dimorphic pathways in autism spectrum disorders. Nature communications. 2016;7:10717.

25. Hulbert SW, Bey AL, Jiang YH. Environmental enrichment has minimal effects on behavior in the Shank3 complete knockout model of autism spectrum disorder. Brain behavior. 2018;8(11):e01107.

26. Janssen H, Bernhardt J, Collier JM, Sena ES, McElduff P, Attia J, et al. An enriched environment improves sensorimotor function post-ischemic stroke. Neurorehabilit Neural Repair. 2010;24(9):80213.

27. Stamenkovic V, Stamenkovic S, Jaworski T, Gawlak M, Jovanovic M, Jakovcevski I, et al. The extracellular matrix glycoprotein tenascin- $C$ and matrix metalloproteinases modify cerebellar structural plasticity by exposure to an enriched environment. Brain Structure Function. 2017;222(1):393-415.

28. Xie H, Wu Y, Jia J, Liu G, Zhang Q, Yu K, et al. Enrichment-induced exercise to quantify the effect of different housing conditions: a tool to standardize enriched environment protocols. Behav Brain Res. 2013;249:81-9.

29. Kempermann G. Environmental enrichment, new neurons and the neurobiology of individuality. Nature reviews Neuroscience. 2019;20(4):235-45.

30. Boso M, Emanuele E, Minazzi V, Abbamonte M, Politi P. Effect of long-term interactive music therapy on behavior profile and musical skills in young adults with severe autism. Journal of alternative complementary medicine (New York NY). 2007;13(7):709-12.

31. Kandalaft MR, Didehbani N, Krawczyk DC, Allen TT, Chapman SB. Virtual reality social cognition training for young adults with high-functioning autism. J Autism Dev Disord. 2013;43(1):34-44.

32. Mukherjee SB. Autism Spectrum Disorders - Diagnosis and Management. Indian J Pediatr. 2017;84(4):307-14.

33. Woo CC, Leon M. Environmental enrichment as an effective treatment for autism: a randomized controlled trial. Behavioral neuroscience. 2013;127(4):487-97.

34. Carlson GC. Glutamate receptor dysfunction and drug targets across models of autism spectrum disorders. Pharmacol Biochem Behav. 2012;100(4):850-4.

35. Chakrabarti B, Persico A, Battista N, Maccarrone M. Endocannabinoid Signaling in Autism. Neurotherapeutics: the journal of the American Society for Experimental NeuroTherapeutics. 2015;12(4):837-47. 
36. Kerr DM, Downey L, Conboy M, Finn DP, Roche M. Alterations in the endocannabinoid system in the rat valproic acid model of autism. Behav Brain Res. 2013;249:124-32.

37. Rojas DC. The role of glutamate and its receptors in autism and the use of glutamate receptor antagonists in treatment. Journal of neural transmission (Vienna, Austria: 1996). 2014;121(8):891905.

38. Silverman JL, Tolu SS, Barkan CL, Crawley JN. Repetitive self-grooming behavior in the BTBR mouse model of autism is blocked by the mGluR5 antagonist MPEP. Neuropsychopharmacology: official publication of the American College of Neuropsychopharmacology. 2010;35(4):976-89.

39. Gould GG, Seillier A, Weiss G, Giuffrida A, Burke TF, Hensler JG, et al. Acetaminophen differentially enhances social behavior and cortical cannabinoid levels in inbred mice. Prog Neuropsychopharmacol Biol Psychiatry. 2012;38(2):260-9.

40. McFarlane HG, Kusek GK, Yang M, Phoenix JL, Bolivar VJ, Crawley JN. Autism-like behavioral phenotypes in BTBR T+tf/J mice. Genes Brain Behav. 2008;7(2):152-63.

41. Meyza KZ, Defensor EB, Jensen AL, Corley MJ, Pearson BL, Pobbe RL, et al. The BTBR T+tf/J mouse model for autism spectrum disorders-in search of biomarkers. Behav Brain Res. 2013;251:25-34.

42. Vandekar S, Tao R, Blume J. A Robust Effect Size Index. Psychometrika. 2020;85(1):232-46.

\section{Figures}

\section{Figure 1}

Expression of NMDA1 and CB1 receptors in the cerebellar cortex of C57BL/6 (C57) and BTBR+Itpr3tf/J (BT) mice raised in standard environments. (A) Fluorescent signal of NMDA1 receptors (red). (B) Images corresponding to the squares in A. (C) Images obtained from averaging the fluorescence of a Z-stack of 10 images separated by $1 \mu \mathrm{m}$. (D) images corresponding to the squares in $C$. The contours emphasize the shape of Purkinje cell somas and dendrites. $(E-H)$ As in A-F for the expression of CB1 receptors (green). H) The contours emphasize the CB1R presynaptic terminals around Purkinje cell soma. ML, Molecular cell layer, PCL, Purkinje cell layer; GCL, Granule cell layer. All slices were stained with DAPI and identically processed (blue). 


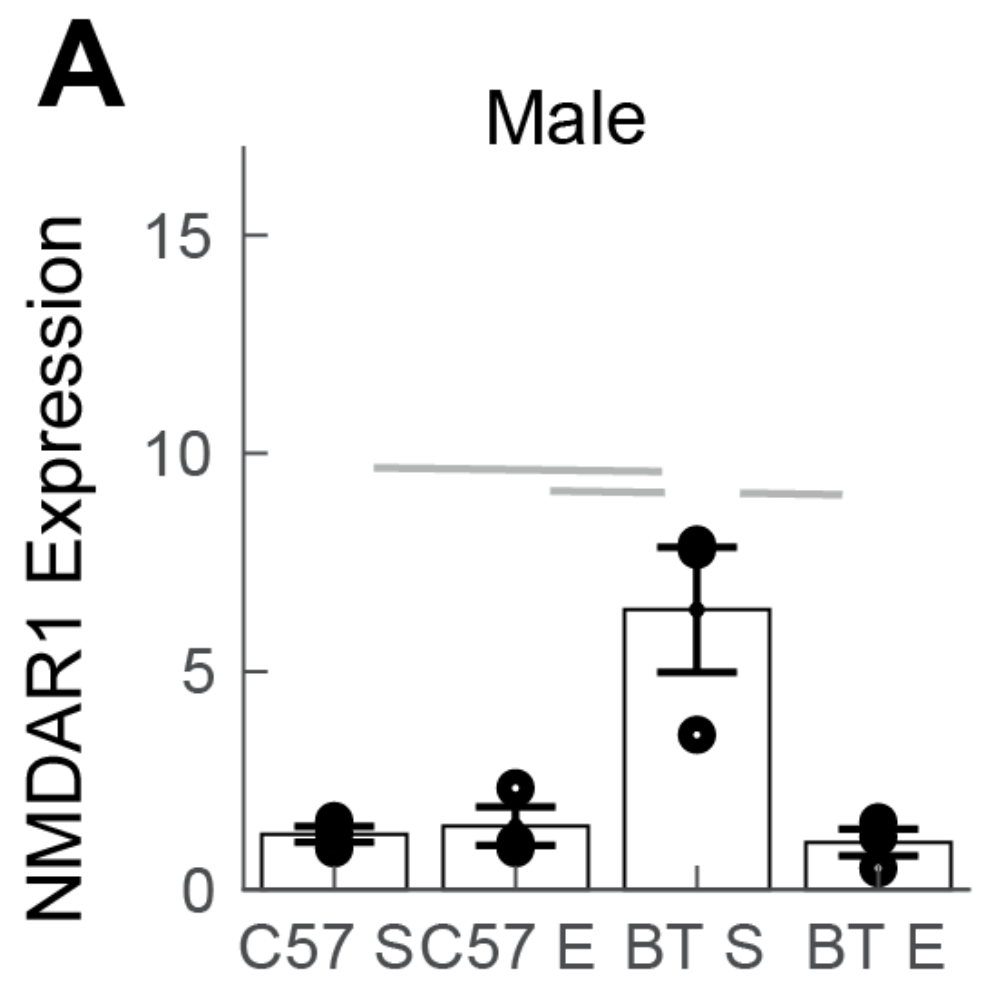

B
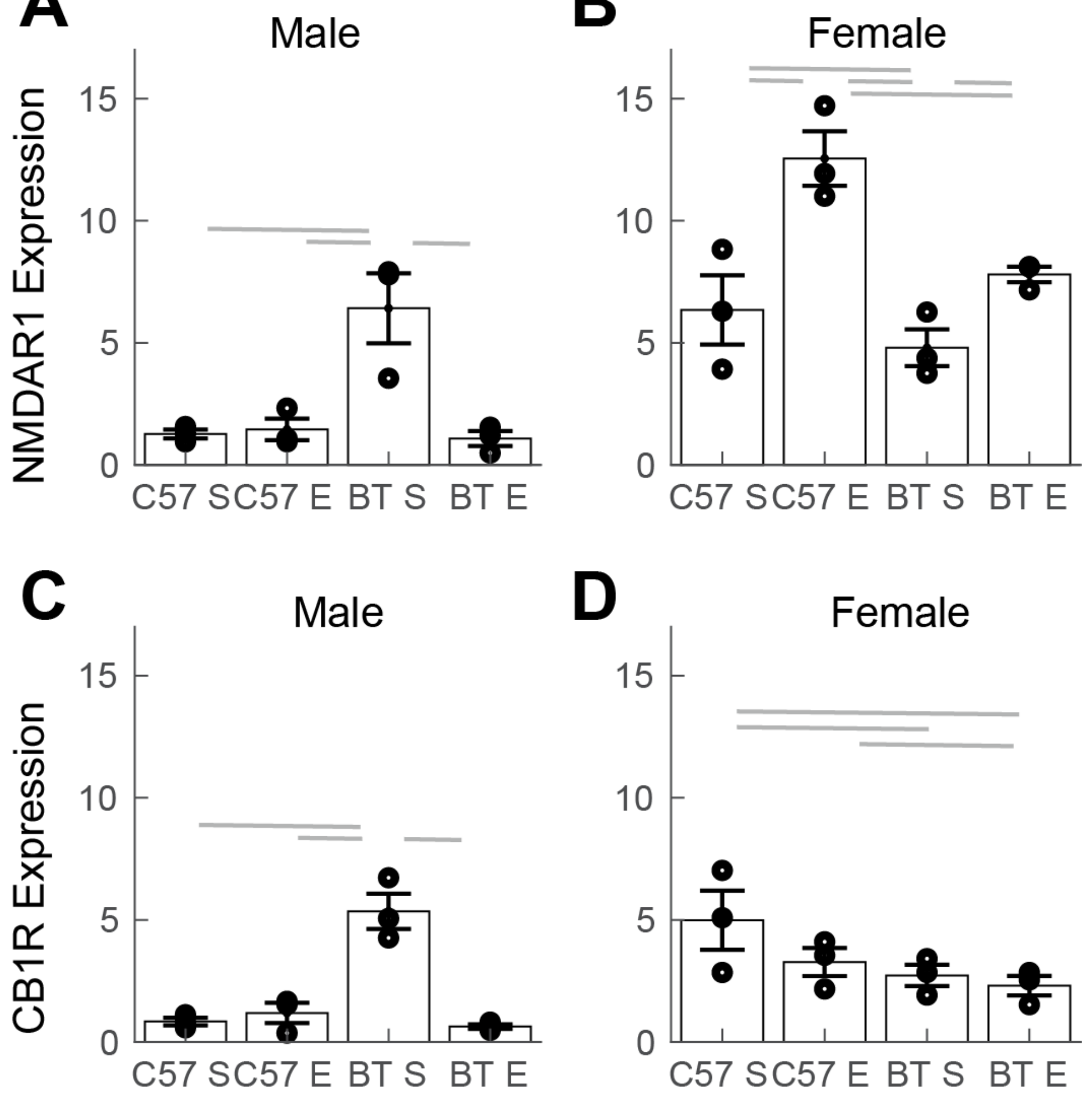

Figure 2

Effects of environmental enrichment on the expression of glutamate (NMDAR1) and endocannabinoid (CB1R) receptors in C57BL/6 (C57) and BTBR+Itpr3tf/J (BT) mice in the cerebellar cortex. (A) Values of NMDAR1/DAPI. The symbols correspond to the average expression of the receptor in each animal. Animal groups were from each genetic background (C57 or BT) exposed to standard (S) or enriched (E) environments. Each column shows mean and SEM. The horizontal lines indicate statistical significance 
$(p<0.05)$. (B) The same analysis as in A for female mice. (C-D) as in A and B for the expression of CB1R. Each data point is the average of 3 samples obtained from the same mice. We collected tissue from 3 animals for each experimental condition.

\section{Supplementary Files}

This is a list of supplementary files associated with this preprint. Click to download.

- AuthorChecklistE10only.pdf

- SuplementaryMaterials.pdf 